\title{
Pointwise Inequalities in Variable Sobolev Spaces and Applications
}

\author{
Alexandre Almeida and Stefan Samko
}

\begin{abstract}
Pointwise estimates for variable exponent Sobolev functions are derived to obtain several results on Sobolev spaces with variable exponent. Hypersingular operators acting in these spaces are considered and the corresponding boundedness and pointwise statements are given over bounded open sets with Lipschitz boundary. Moreover, classical Sobolev embeddings into Hölder spaces are generalized to the variable exponent setting.
\end{abstract}

Keywords. Variable exponent, Sobolev space, Sobolev embedding, Hölder space, variable order, maximal function, hypersingular integral

Mathematics Subject Classification (2000). 46E35, 26D10, 26A33

\section{Introduction}

Lebesgue and Sobolev spaces with variable exponent have been intensively studied during the last years by many authors. We only refer to the papers [27, 38], where the basics of such spaces were developed, to the papers [12, 33], where the denseness of nice functions in variable Sobolev spaces was considered, and to the papers $[7,8,10,11,25,26,29,34,35]$ and the recent preprints $[5,6]$ and references therein, where several results on maximal, potential and singular operators in variable Lebesgue spaces were obtained. We also mention the survey [37].

Roughly speaking, the interest in variable exponent spaces comes not only from their mathematical curiosity but also from their relevance in many applications such as fluid dynamics, elasticity theory, differential equations with non-standard growth conditions and image restoration (cf. [11, 28, 32]).

A. Almeida: Departamento de Matemática, Universidade de Aveiro, 3810-193 Aveiro, Portugal; alexandre@mat.ua.pt

S. Samko: Faculdade de Ciências e Tecnologia, Universidade do Algarve, Campus de Gambelas, 8005-139 Faro, Portugal; ssamko@ualg.pt 
In this paper we deal with pointwise type inequalities in Sobolev spaces with variable exponent. We recover the well-known statement that the oscillation of Sobolev functions may be estimated in terms of the fractional maximal function of its gradient (see, for instance, $[4,19,20,23]$ ), and use it to study Sobolev embeddings into Hölder spaces with variable order and hypersingular integrals of variable Sobolev functions.

Sobolev embeddings on variable exponent Sobolev spaces have been studied by many authors, mainly in the case when the exponent is less than the dimension of the Euclidean space $\mathbb{R}^{n}$ (see $[10,13,14,15,30]$ ). The case when the exponent is greater than $n$ was less studied. We refer first of all to [13], where embeddings into the Hölder spaces with variable exponent have been obtained (see Theorems 5.4 and 5.5 in [13]), and to [21] where the capacity approach was used to get embeddings into the space of continuous functions or in $L^{\infty}(\Omega)$.

In this paper, we prove a slightly different version of the embeddings into Hölder spaces with variable exponents, obtained in [13], by following another approach (see Theorem 4.7 below). In our proof we base ourselves on estimation of the oscillation of $f \in W^{1, p(\cdot)}(\Omega)$ by fractional maximal functions of $\nabla f$ developed for instance in $[19,20]$.

We also consider hypersingular integrals of functions from Sobolev spaces with variable exponent defined over bounded open sets. We derive boundedness and pointwise results for the hypersingular operator of variable order $\alpha=\alpha(x)$ acting in $W^{1, p(\cdot)}(\Omega)$ into an appropriate variable Lebesgue space, in the case when $\Omega$ has Lipschitz boundary. The results obtained are new, even in the particular case when the exponents are constant. We point out that hypersingular integrals were recently studied on variable exponent spaces in the papers [2] and [3]. For a detailed discussion on general hypersingular integrals and their applications see [36].

The paper is structured as follows. After some necessary preliminaries, in Section 3 we generalize some known pointwise estimates to the variable Sobolev spaces. Sobolev embeddings are studied in Section 4, where the main result is formulated in Theorem 4.4. Finally, Section 5 is devoted to the study of hypersingular integrals of Sobolev functions defined over bounded open sets. As mentioned above, boundedness results are given and pointwise convergence is discussed.

\section{Preliminaries}

The notation we will follow is standard or it will be properly introduced whenever needed. Everywhere below, $\Omega$ is assumed to be a non-empty open set of the Euclidean space $\mathbb{R}^{n}$. 
2.1. On variable Lebesgue spaces. Let $p: \Omega \rightarrow[1, \infty)$ be a (Lebesgue) measurable bounded function. Put

$$
\bar{p}_{\Omega}:=\sup _{x \in \Omega} p(x) \text { and } \quad \underline{p}_{\Omega}:=\inf _{x \in \Omega} p(x) .
$$

In the sequel we always assume that $1<\underline{p}_{\Omega} \leq \bar{p}_{\Omega}<\infty$. By $L^{p(\cdot)}(\Omega)$ we denote the space of all measurable functions $f$ on $\Omega$ such that the modular

$$
\mathfrak{I}_{p(\cdot), \Omega}(f):=\int_{\Omega}|f(x)|^{p(x)} d x
$$

is finite. This space, sometimes called Lebesgue space with variable exponent or variable Lebesgue space, is a Banach space with respect to the norm

$$
\|f\|_{p(\cdot), \Omega}:=\inf \left\{\lambda>0: \mathfrak{I}_{p(\cdot), \Omega}\left(\frac{f}{\lambda}\right) \leq 1\right\}, \quad f \in L^{p(\cdot)}(\Omega)
$$

When $p(x) \equiv p$ is constant then $L^{p(\cdot)}(\Omega)$ coincides with the standard Lebesgue space $L^{p}(\Omega)$. Details on variable Lebesgue spaces may be found in the papers $[12,16,27,35,38]$. We stress that some basic properties of the classic Lebesgue spaces are not transferred to the variable exponent case. For instance, the space $L^{p(\cdot)}(\Omega)$ is no longer translation invariant. As a consequence, Young's theorem and the so called mean continuity property fail in general (see [17] and [35] for details). An important property of the variable Lebesgue space is that the convergence in norm is equivalent to the modular convergence: given $\left\{f_{k}\right\}_{k \in \mathbb{N}_{0}} \subset L^{p(\cdot)}(\Omega)$, then $\left\|f_{k}\right\|_{p(\cdot), \Omega} \rightarrow 0$ if and only if $\mathfrak{I}_{p(\cdot), \Omega}\left(f_{k}\right) \rightarrow 0$, as $k \rightarrow \infty$.

As in the classic case, one also defines the Sobolev space of variable exponent $W^{1, p(\cdot)}(\Omega)$ as the space of all functions $f \in L^{p(\cdot)}(\Omega)$ for which the first order derivatives also belong to $L^{p(\cdot)}(\Omega)$. $W^{1, p(\cdot)}(\Omega)$ is a Banach space equipped with the norm

$$
\|f\|_{1, p(\cdot), \Omega}:=\|f\|_{p(\cdot), \Omega}+\|\mid \nabla f\|_{p(\cdot), \Omega}, \quad f \in W^{1, p(\cdot)}(\Omega),
$$

where $\nabla f$ denotes the (weak) gradient of $f$. In order to emphasize that we are dealing with variable exponents, we shall write $p(\cdot)$ instead of $p$ to denote an exponent function. For simplicity, when $\Omega=\mathbb{R}^{n}$ then we will omit the $\Omega$ in the notation: for example, we only write $\|\cdot\|_{p(\cdot)}$ instead of $\|\cdot\|_{p(\cdot), \mathbb{R}^{n}}$ to denote the norm (1).

We write $B(x, r)$ for an open ball in $\mathbb{R}^{n}$ centered at $x \in \mathbb{R}^{n}$ and of radius $r>0$, and we denote its measure by $|B(x, r)|$.

The fractional maximal function $\mathcal{M}_{\Omega}^{\lambda(\cdot)} g, 0 \leq \lambda(x)<n$, of a locally integrable function $g$ is given by

$$
\mathcal{M}_{\Omega}^{\lambda(\cdot)} g(x)=\sup _{r>0} \frac{1}{|B(x, r)|^{1-\frac{\lambda(x)}{n}}} \int_{B(x, r) \cap \Omega}|g(y)| d y, \quad x \in \Omega .
$$


Fractional maximal functions of variable order within the variable exponent spaces were considered in [24]. If $\lambda(x) \equiv 0$, then $\mathcal{M}_{\Omega}^{0}=\mathcal{M}_{\Omega}$ is the usual Hardy-Littlewood maximal operator. We shall denote by $\mathcal{P}(\Omega)$ the class of all exponents $p(\cdot)$, such that $\mathcal{M}_{\Omega}$ is bounded in $L^{p(\cdot)}(\Omega)$.

We will often assume the log-Hölder continuity condition

$$
|p(x)-p(y)| \leq \frac{A_{0}}{\ln \frac{1}{|x-y|}}, \quad x, y \in \Omega, \quad|x-y| \leq \frac{1}{2} .
$$

Note that (2) implies

$$
|p(x)-p(y)| \leq \frac{2 N A_{0}}{\ln \frac{2 N}{|x-y|}}, \quad x, y \in \Omega, \quad|x-y| \leq N
$$

where $N \in \mathbb{N}$. Diening [8] proved that if $\Omega$ is bounded and $p(\cdot)$ satisfies $(2)$, then $p(\cdot) \in \mathcal{P}(\Omega)$. The boundedness of $\mathcal{M}_{\Omega}$ over unbounded open sets holds if $p(\cdot)$ fulfills $(2)$ and has a logarithmic decay at infinity (see [7]),

$$
|p(x)-p(\infty)| \leq \frac{A_{\infty}}{\ln (e+|x|)}, \quad x \in \Omega .
$$

According to the result on the $(p(\cdot) \rightarrow q(\cdot))$-boundedness of the Riesz potential operator,

$$
I_{\Omega}^{\beta(\cdot)} g(x)=\int_{\Omega} \frac{g(y) d y}{|x-y|^{n-\beta(x)}},
$$

obtained in [34] and the boundedness of the maximal operator proved in [8], the following theorem is valid over bounded open sets in $\mathbb{R}^{n}$.

Theorem 2.1. Let $p(\cdot)$ satisfy $(2)$ and $\beta(\cdot)$ satisfy the conditions

$$
\inf _{x \in \Omega} \beta(x)>0, \quad \sup _{x \in \Omega} \beta(x) p(x)<n,
$$

and let $\frac{1}{q(x)}=\frac{1}{p(x)}-\frac{\beta(x)}{n}$. Then

$$
\left\|I_{\Omega}^{\beta(\cdot)} g\right\|_{q(\cdot), \Omega} \leq C\|g\|_{p(\cdot), \Omega}
$$

Theorem $2.2([24])$. Let $p(\cdot)$ satisfy $(2)$ and $\beta(\cdot)$ satisfy the conditions

$$
\inf _{x \in \Omega} \beta(x)>0, \quad \sup _{x \in \Omega} \beta(x) p(x)<n,
$$

and let $\frac{1}{q(x)}=\frac{1}{p(x)}-\frac{\beta(x)}{n}$. Then

$$
\left\|\mathcal{M}_{\Omega}^{\beta(\cdot)} g\right\|_{q(\cdot), \Omega} \leq C\|g\|_{p(\cdot), \Omega} .
$$


Observe that the statement (3) follows from the well-known pointwise estimate

$$
\mathcal{M}_{\Omega}^{\beta(\cdot)} g(x) \leq c I_{\Omega}^{\beta(\cdot)}(|g|)(x), \quad x \in \Omega
$$

(see $[1$, p. 72$]$ ), $I_{\Omega}^{\beta(\cdot)}$ being the Riesz potential operator of variable order, and from the Sobolev-type Theorem 2.1.

2.2. Hölder spaces of variable order. Hölder spaces have an important role in the study of regularity properties in the framework of variational calculus and differential equations. We consider here a natural generalization as follows.

Let $B C(\Omega)$ be the class of bounded continuous functions on $\Omega$. For a measurable function $\alpha: \Omega \rightarrow(0,1]$ and $f \in B C(\Omega)$, let

$$
[f]_{\alpha(\cdot), \Omega}:=\sup _{\substack{x, x+h \in \Omega \\ 0<|h| \leq 1}} \frac{|f(x+h)-f(x)|}{|h|^{\alpha(x)}} .
$$

By $C^{0, \alpha(\cdot)}(\Omega)$ we denote the space of all functions $f$ in $B C(\Omega)$ for which $[f]_{\alpha(\cdot), \Omega}$ is finite. $C^{0, \alpha(\cdot)}(\Omega)$ is a Banach space with respect to the norm

$$
\|f\|_{C^{0, \alpha(\cdot)(\Omega)}}=\|f\|_{\infty, \Omega}+[f]_{\alpha(\cdot), \Omega} .
$$

This is a generalization of the standard Hölder spaces $C^{0, \alpha}(\Omega)$ with constant $\alpha \in(0,1]$. As in the case of the variable Lebesgue spaces, we shall write $\alpha(\cdot)$ instead of $\alpha$ to emphasize that we are dealing with a variable order of regularity.

Hölder spaces with variable order were considered in [22, 31], where the mapping properties of fractional integration operators in such spaces were studied.

\section{Pointwise inequalities for Sobolev functions}

It is known that oscillation of functions in Sobolev spaces can be estimated in terms of the fractional maximal function of its gradient; see for instance $[4,19$, 20, 23], where such estimates of the oscillation were used to derive important properties of functions in Sobolev spaces within the classical setting. This will be extended to the case of variable exponents in the next section. In this section we recall the above mentioned estimates of the oscillation (see Proposition 3.3) and give it with proofs for completeness of presentation.

First we observe that the following statements are valid.

Lemma 3.1 ([18], Lemma 7.16). Let $B$ be a ball in $\mathbb{R}^{n}$. If $g \in W^{1,1}(B)$, then

$$
\left|g(x)-g_{B}\right| \leq c(n) \int_{B} \frac{|\nabla g(z)|}{|x-z|^{n-1}} d z
$$

almost everywhere in $B$, where $g_{B}:=\frac{1}{|B|} \int_{B} g(z) d z$ denotes the average of $g$ over $B$. 
Lemma 3.2. Let $D \subset \mathbb{R}^{n}$ be an open bounded set, $0<\alpha \leq n$ and $0 \leq \lambda<\alpha$. Then there exists $c>0$, not depending on $f, x$ and $\lambda$, such that

$$
\int_{D} \frac{|f(z)| d z}{|x-z|^{n-\alpha}} \leq \frac{c}{\alpha-\lambda}(\operatorname{diam}(D))^{\alpha-\lambda} \mathcal{M}_{D}^{\lambda} f(x)
$$

for almost all $x \in D$, and for every $f \in L^{1}(D)$, and it is admitted that $\lambda$ may depend on $x$.

Proof. Let $d=\operatorname{diam}(D)$. We have

$$
\begin{aligned}
\int_{D} \frac{|f(z)| d z}{|x-z|^{n-\alpha}} & =\sum_{k=0}^{\infty} \int_{D \cap\left(B\left(x, \frac{d}{2^{k}}\right) \backslash B\left(x, \frac{d}{2^{k+1}}\right)\right)} \frac{|f(z)|}{|x-z|^{n-\alpha}} d z \\
& \leq \sum_{k=0}^{\infty}\left(\frac{2^{k+1}}{d}\right)^{n-\alpha} \int_{D \cap B\left(x, \frac{d}{2^{k}}\right)}|f(z)| d z \\
& \leq c(n) \sum_{k=0}^{\infty}\left(\frac{2^{k}}{d}\right)^{\lambda-\alpha} \mathcal{M}_{D}^{\lambda} f(x),
\end{aligned}
$$

from which (4) follows.

Proposition 3.3. Let $\Omega$ be a bounded open set with Lipschitz boundary or let $\Omega=\mathbb{R}^{n}$. Then for every $f \in W_{\text {loc }}^{1,1}(\Omega)$ and almost all $x, y \in \Omega$ there holds

$$
|f(x)-f(y)| \leq c\left[\frac{|x-y|^{1-\lambda}}{1-\lambda} \mathcal{M}_{\Omega}^{\lambda}(|\nabla f|)(x)+\frac{|x-y|^{1-\mu}}{1-\mu} \mathcal{M}_{\Omega}^{\mu}(|\nabla f|)(y)\right],
$$

where $\lambda, \mu \in[0,1)$ and the constant $c>0$ does not depend on $f, x, y, \lambda, \mu$ and $\Omega$, and it is admitted that $\lambda$ and $\mu$ may depend on $x$ and $y$.

Proof. For bounded domains estimate (5) can be proved as in [20, Lemma 4]. For $\Omega=\mathbb{R}^{n}$ the arguments are similar: we observe that for all $x, y \in \mathbb{R}^{n}, x \neq y$, there exists a ball $B_{x, y}$ containing these points such that $\operatorname{diam}\left(B_{x, y}\right) \leq 2|x-y|$. Then we write $|f(x)-f(y)| \leq\left|f(x)-f_{B_{x, y}}\right|+\left|f(y)-f_{B_{x, y}}\right|$ and it remains to make use of Lemma 3.1 and afterwards Lemma 3.2 with $\alpha=1$.

\section{Sobolev embeddings with variable exponent}

The main statement of this section is Theorem 4.4, which shows that functions in $W^{1, p(\cdot)}(\Omega)$ are Hölder continuous everywhere where $p(x)>n$. First we need some auxiliary statements. 
Lemma $4.1([10])$. Let $p: \mathbb{R}^{n} \rightarrow[1, \infty)$ be a continuous exponent. Then $p(\cdot)$ satisfies the log-Hölder condition (2) if and only if there exists a constant $C>0$ such that

$$
|B|^{\inf _{z \in B} \frac{1}{p(z)}-\sup _{z \in B} \frac{1}{p(z)}} \leq C,
$$

for all open balls $B$ in $\mathbb{R}^{n}$.

Let $B$ be a ball in $\mathbb{R}^{n}$ and let $p(\cdot)$ be a bounded exponent. For simplicity, we will denote by $\frac{1}{p_{B}}$ the average of the function $\frac{1}{p}$ over $B$, that is

$$
\frac{1}{p_{B}}:=\frac{1}{|B|} \int_{B} \frac{d z}{p(z)}
$$

Lemma $4.2([10])$. Let $p(\cdot) \in \mathcal{P}\left(\mathbb{R}^{n}\right)$. Then for every ball $B$ there holds

$$
\left\|\chi_{B}\right\|_{p(\cdot)} \leq C(p)|B|^{\frac{1}{p_{B}}} .
$$

We will also make use of the following statement in which

$$
\Pi_{p, \Omega}:=\{x \in \Omega: p(x)>n\} .
$$

Lemma 4.3. Let $\Omega$ be a bounded domain and let $f \in L^{p(\cdot)}(\Omega)$, where $p(\cdot)$ satisfies condition (2). Assume also that the set $\Pi_{p, \Omega}$ is non-empty. Then

$$
\mathcal{M}_{\Omega}^{\frac{n}{p(x)}} f(x) \leq c\|f\|_{p(\cdot), \Omega}, \quad x \in \Pi_{p, \Omega},
$$

with $c>0$ not depending on $x$ nor $f$.

Proof. First we observe that the exponent $p(\cdot)$ may be extended to the whole space $\mathbb{R}^{n}$ with the preservation of its continuity modulus. In fact, since $p(\cdot)$ is uniformly continuous (and bounded) on $\Omega$, then it extends to a continuous function on $\bar{\Omega}$. By a known extension result described in [39], Chapter 6 , Section 2, there exists an extension $\tilde{p}: \mathbb{R}^{n} \rightarrow[1, \infty)$ satisfying a corresponding condition to $(2)$ on $\mathbb{R}^{n}$ (possibly with a different constant). From $\tilde{p}(\cdot)$ we may construct another extension $\tilde{\tilde{p}}(\cdot): \mathbb{R}^{n} \rightarrow[1, \infty)$, also preserving the continuity modulus, in such a way that $\widetilde{p}(\cdot)$ is constant outside some large ball (see [10], Theorem 4.2 and Corollary 4.3, for details). In particular, we have $\widetilde{\tilde{p}}(\cdot) \in \mathcal{P}\left(\mathbb{R}^{n}\right)$.

Let $B=B(x, r)$ be any ball centered at $x \in \Pi_{p, \Omega}$. By the Hölder inequality we obtain

$$
\frac{1}{|B|^{1-\frac{1}{p(x)}}} \int_{B \cap \Omega}|f(z)| d z \leq \frac{c(p)}{|B|^{1-\frac{1}{p(x)}}}\|f\|_{\tilde{\tilde{p}}(\cdot)}\left\|\chi_{B}\right\|_{\tilde{\tilde{p}}^{\prime}(\cdot)},
$$

where $\widetilde{\tilde{p}}^{\prime}(\cdot)$ is the usual conjugate exponent, $\frac{1}{\tilde{\tilde{p}}(\cdot)}+\frac{1}{\tilde{\tilde{p}}^{\prime}(\cdot)}=1$, and it is assumed that $f$ is continued as zero beyond $\Omega$. 
Since $\widetilde{\tilde{p}}(\cdot) \in \mathcal{P}\left(\mathbb{R}^{n}\right)$, then the maximal operator is also bounded in $L^{\widetilde{\tilde{p}}^{\prime}(\cdot)}\left(\mathbb{R}^{n}\right)$ (see $\left[9\right.$, Lemma 8.1]). Therefore, Lemma 4.2 is applicable which yields $\left\|\chi_{B}\right\|_{\tilde{\tilde{p}}^{\prime}(\cdot)} \leq$ $c_{1}(p)|B|^{\frac{1}{\tilde{p}_{B}^{\prime}}}$. Hence,

$$
\frac{1}{|B|^{1-\frac{1}{p(x)}}} \int_{B \cap \Omega}|f(z)| d z \leq c\|f\|_{p(\cdot), \Omega}|B|^{\frac{1}{p(x)}-\frac{1}{\tilde{\tilde{p}}_{B}}}
$$

If $|B| \leq 1$ then Lemma 4.1 provides the estimate $|B|^{\frac{1}{p(x)}-\frac{1}{\tilde{\tilde{p}}_{B}}} \leq C$, for some $C>0$ independent of $B$. Suppose now that $|B|>1$. Notice that if $r>\operatorname{diam}(\Omega)$ then $|B|>|\Omega|$, so that

$$
\frac{1}{|B|^{1-\frac{1}{p(x)}}} \int_{B \cap \Omega}|f(z)| d z \leq \frac{1}{|\Omega|^{1-\frac{1}{p(x)}}} \int_{\Omega}|f(z)| d z \leq c(\Omega)\|f\|_{p(\cdot), \Omega} .
$$

Hence, only the case $r \leq \operatorname{diam}(\Omega)$ is of interest according to our purposes. In that case, the right-hand side in (8) may be estimated as follows:

$$
|B|^{\frac{1}{p(x)}-\frac{1}{\tilde{\tilde{p}}_{B}}} \leq|B|^{1-(\overline{\tilde{\tilde{p}}})^{-1}} \leq C \operatorname{diam}(\Omega)^{n} .
$$

This completes the proof of (7).

Now we are able to give an important pointwise inequality.

Theorem 4.4. Let $\Omega$ be a bounded open set with Lipschitz boundary and suppose that $p(\cdot)$ satisfies the local logarithmic condition (2) and has a non-empty set $\Pi_{p, \Omega}$. If $f \in W^{1, p(\cdot)}(\Omega)$, then

$$
|f(x)-f(y)| \leq C(x, y)\||\nabla f|\|_{p(\cdot), \Omega}|x-y|^{1-\frac{n}{\min [p(x), p(y)]}}
$$

for all $x, y \in \Pi_{p, \Omega}$ such that $|x-y| \leq 1$, where

$$
C(x, y)=\frac{c}{\min [p(x), p(y)]-n}
$$

with $c>0$ not depending on $f, x$ and $y$.

Proof. After modifying $f$ on a set of zero measure, we make use of (5) with $\lambda=\frac{n}{p(x)} \in(0,1)$ and $\mu=\frac{n}{p(y)} \in(0,1)$ and get

$$
\begin{aligned}
\mid f(x) & -f(y) \mid \\
& \leq \frac{c|x-y|^{1-\frac{n}{\min [p(x), p(y)]}}}{\min [p(x)-n, p(y)-n]}\left[\mathcal{M}_{\Omega}^{\frac{n}{p(x)}}(|\nabla f|)(x)+\mathcal{M}_{\Omega}^{\frac{n}{p(y)}}(|\nabla f|)(y)\right]
\end{aligned}
$$

for all $x, y \in \Pi_{p, \Omega}$. Hence, (9) immediately follows from (7). 
Remark 4.5. Let $D$ be a subset in $\Pi_{p, \Omega}$. Under the assumption $\inf _{x \in D} p(x)>n$, one may take a constant in (9) not depending on $x, y$ when $x$ and $y$ run the set $D$. In particular, if $\underline{p}_{\Omega}>n$, estimate (9) is valid for the whole $\Omega$ with an absolute constant.

Corollary 4.6. Let $\Omega$ be a bounded open set with Lipschitz boundary and let $p(\cdot)$ be under the assumptions of Theorem 4.4. If $f \in W^{1, p(\cdot)}(\Omega)$, then the estimate (9) may be written in the form

$$
|f(x)-f(x+h)| \leq \frac{c}{\min [p(x), p(x+h)]-n}\||\nabla f|\|_{p(\cdot), \Omega}|h|^{1-\frac{n}{p(x)}},
$$

where $x, x+h \in \Pi_{p, \Omega}$ and $|h| \leq 1$, with $c>0$ not depending on $x$, $h$, and $f$.

Proof. Indeed, it suffices to observe that for $x$ and $y$ belonging to a bounded set we have

$$
|x-y|^{\frac{n}{p(x)}} \sim|x-y|^{\frac{n}{p(y)}}
$$

thanks to the log-condition for $p(\cdot)$.

Theorem 4.4 suggests that functions in $W^{1, p(\cdot)}(\Omega)$ admit a Hölder continuous representative of variable order.

Theorem 4.7. Let $\Omega$ be a bounded open set and suppose that $p(\cdot)$ satisfies the logarithmic condition (2). If $\inf _{x \in \Omega} p(x)>n$, then the estimate

$$
|f(x)| \leq C\left[\frac{\|f\|_{p(\cdot), \Omega}}{[\operatorname{dist}(x, \partial \Omega)]^{\frac{n}{p(x)}}}+\||\nabla f|\|_{p(\cdot), \Omega}\right]
$$

is valid, with $C>0$ independent of $x \in \Omega$ and $f \in W^{1, p(\cdot)}(\Omega)$. If, in addition, $\Omega$ has Lipschitzian boundary, then we have

$$
W^{1, p(\cdot)}(\Omega) \hookrightarrow C^{0,1-\frac{n}{p(\cdot)}}(\Omega),
$$

where “œ" means continuous embedding.

Proof. Fix $x \in \Omega$ and let $B_{x}$ be a ball containing $x$. According to Lemma 3.1, estimate (4) with $\alpha=1$ and $\lambda=\frac{n}{p(x)}$, and inequality (7), for $f \in W^{1, p(\cdot)}(\Omega)$ we have

$$
\begin{aligned}
\left|f(x)-f_{B_{x}}\right| & \leq c \operatorname{diam}\left(B_{x}\right)^{1-\frac{n}{p(x)}} \mathcal{M}_{\Omega}^{\frac{n}{p(x)}}(|\nabla f|)(x) \\
& \leq c \operatorname{diam}\left(B_{x}\right)^{1-\frac{n}{p(x)}}\||\nabla f|\|_{p(\cdot), \Omega},
\end{aligned}
$$

where it is assumed that the radius $r$ of the ball $B_{x}$ is sufficiently small, say $r=\frac{1}{2} \operatorname{dist}(x, \partial \Omega)$. For $f_{B_{x}}$ we may proceed as in the proof of Lemma 4.3. Hence, the Hölder inequality combined with (6) yield the estimate

$$
\left|f_{B_{x}}\right| \leq c(p)\left|B_{x}\right|^{-\frac{1}{p_{B x}}}\|f\|_{p(\cdot), \Omega}
$$


Since $\left|B_{x}\right|^{-\frac{1}{p_{B}}} \leq c\left|B_{x}\right|^{-\frac{1}{p(x)}}$, we also have

$$
\left|f_{B_{x}}\right| \leq c(p)\left|B_{x}\right|^{-\frac{1}{p(x)}}\|f\|_{p(\cdot), \Omega} .
$$

Thus, having in mind the value of $r$ above, we arrive at (11) from (13) and (14).

If $\Omega$ has Lipschitz boundary, then we may derive the embedding

$$
W^{1, p(\cdot)}(\Omega) \hookrightarrow L^{\infty}(\Omega)
$$

In fact, in that case, it is known (see [10]) that there exists a bounded linear extension operator

$$
\mathcal{E}: W^{1, p(\cdot)}(\Omega) \rightarrow W^{1, \tilde{\tilde{p}}(\cdot)}\left(\mathbb{R}^{n}\right)
$$

such that $\left.\mathcal{E} f\right|_{\Omega}=f$ almost everywhere, where $\widetilde{\tilde{p}}(\cdot)$ is the extension of $p(\cdot)$ used in the proof of Lemma 4.3. Similarly to (13), there holds

$$
\left|f(x)-\mathcal{E} f_{B_{x}}\right| \leq c \operatorname{diam}\left(B_{x}\right)^{1-\frac{n}{p(x)}}|||\nabla f| \|_{p(\cdot), \Omega},
$$

where now we suppose that the ball $B_{x}$ is arbitrary (containing $x$ ). Moreover, we have

$$
\left|\mathcal{E} f_{B_{x}}\right| \leq c\left|B_{x}\right|^{-\frac{1}{\tilde{\tilde{p}} B_{x}}}\|\mathcal{E} f\|_{\tilde{\tilde{p}}(\cdot)} \leq c_{1}\left|B_{x}\right|^{-\frac{1}{\tilde{p}_{B x}}}\|f\|_{p(\cdot), \Omega}
$$

Taking a ball such that $\left|B_{x}\right|=1$, we get

$$
|f(x)| \leq\left|f(x)-f_{B_{x}}\right|+\left|f_{B_{x}}\right| \leq C(p)\|f\|_{1, p(\cdot), \Omega},
$$

which implies (15). The embedding (12) follows then from (10) and (15).

In the particular case when the exponent is constant, $p(x) \equiv p>n$, we recover the classical Sobolev embedding.

\section{Hypersingular operators on spaces $\mathrm{W}^{1, p(\cdot)}(\Omega)$}

We consider hypersingular integral operators of variable order $\alpha=\alpha(x)$, $0<\alpha(x)<1, x \in \Omega$, given by

$$
\mathcal{D}^{\alpha(\cdot)} f(x)=\int_{\Omega} \frac{f(x)-f(y)}{|x-y|^{n+\alpha(x)}} d y, \quad x \in \Omega .
$$

A detailed information about hypersingular integrals of functions defined in $\mathbb{R}^{n}$ can be found in [36]. 
Theorem 5.1. Let $0<\alpha_{0} \leq \alpha(x) \leq \alpha_{1}<1$ and let $\Omega$ be a bounded open set with Lipschitz boundary. Assume also that $p(\cdot)$ satisfies (2) and

$$
\sup _{x \in \Omega} p(x)[1-\alpha(x)]<n .
$$

Then the operator $\mathcal{D}^{\alpha(\cdot)}$ is bounded from $W^{1, p(\cdot)}(\Omega)$ into $L^{q(\cdot)}(\Omega)$ for any exponent $q(\cdot), 1<\underline{q}_{\Omega} \leq \bar{q}_{\Omega}<\infty$, such that

$$
\sup _{x \in \Omega}\left[\frac{1}{p(x)}-\frac{1}{q(x)}+\frac{\alpha(x)}{n}\right]<\frac{1}{n},
$$

the latter being equivalent to

$$
\frac{1}{q(x)}=\frac{1}{p(x)}-\frac{\lambda(x)}{n}, \quad \text { where } \sup _{x \in \Omega}[\lambda(x)+\alpha(x)]<1
$$

Proof. We may assume that $q(x) \geq p(x)$ since $\Omega$ is bounded and one has the imbedding $\|f\|_{q(\cdot), \Omega} \leq c\|f\|_{q^{*}(\cdot), \Omega}$ where $q^{*}(x)=\max \{q(x), p(x)\}$.

By Proposition 3.3, we have

$$
\begin{aligned}
\left|\mathcal{D}^{\alpha(\cdot)} f(x)\right| & \leq \int_{\Omega} \frac{|f(x)-f(y)|}{|x-y|^{n+\alpha(x)}} d y \\
& \leq \frac{c}{1-\lambda(x)} \int_{\Omega} \frac{\mathcal{M}_{\Omega}^{\lambda(\cdot)}(|\nabla f|)(x)+\mathcal{M}_{\Omega}^{\lambda(\cdot)}(|\nabla f|)(y)}{|x-y|^{n+\alpha(x)+\lambda(x)-1}} d y
\end{aligned}
$$

for almost all $x \in \Omega$, with $c>0$ not depending on $x$ and $f$, where $\lambda(x)$ may be an arbitrary function such that $0 \leq \lambda(x)<1$.

Put $\beta(x)=1-\alpha(x)-\lambda(x)$. Then $0<\beta(x)<1$ under the choice $\lambda(x)<$ $1-\alpha(x)$. We choose $\lambda(x)$ so that

$$
\lambda(x) \geq 0 \quad \text { and } \quad \sup _{x \in \Omega}[\lambda(x)+\alpha(x)]<1,
$$

which is possible, since $\sup _{x \in \Omega} \alpha(x) \leq \alpha_{1}<1$. Then

$$
\inf _{x \in \Omega} \beta(x)>0 .
$$

We have

$$
\begin{aligned}
\left|\mathcal{D}^{\alpha(\cdot)} f(x)\right| & \leq c \int_{\Omega} \frac{\mathcal{M}_{\Omega}^{\lambda(\cdot)}(|\nabla f|)(x)}{|x-y|^{n-\beta(x)}} d y+c \int_{\Omega} \frac{\mathcal{M}_{\Omega}^{\lambda(\cdot)}(|\nabla f|)(y)}{|x-y|^{n-\beta(x)}} d y \\
& \leq c|\Omega|^{\frac{\beta(x)}{n}} \mathcal{M}_{\Omega}^{\lambda(\cdot)}(|\nabla f|)(x)+c I_{\Omega}^{\beta(\cdot)}\left[\mathcal{M}_{\Omega}^{\lambda(\cdot)}(|\nabla f|)\right](x)
\end{aligned}
$$


where the first term in the last sum follows from the estimation of the Riesz potential of a constant density. Hence

$$
\left\|\mathcal{D}^{\alpha(\cdot)} f\right\|_{q(\cdot), \Omega} \leq c\left\|\mathcal{M}_{\Omega}^{\lambda(\cdot)}(|\nabla f|)\right\|_{q(\cdot), \Omega}+c\left\|I_{\Omega}^{\beta(\cdot)}\left[\mathcal{M}_{\Omega}^{\lambda(\cdot)}(|\nabla f|)\right]\right\|_{q(\cdot), \Omega} .
$$

By condition (20) and boundedness of $\Omega$, the operator $I_{\Omega}^{\beta(\cdot)}$ is bounded in the space $L^{q(\cdot)}(\Omega)$ so that

$$
\left\|\mathcal{D}^{\alpha(\cdot)} f\right\|_{q(\cdot), \Omega} \leq c\left\|\mathcal{M}_{\Omega}^{\lambda(\cdot)}(|\nabla f|)\right\|_{q(\cdot), \Omega} .
$$

By Theorem 2.2 we then have

$$
\left\|\mathcal{D}^{\alpha(\cdot)} f\right\|_{q(\cdot), \Omega} \leq c\||\nabla f|\|_{p(\cdot), \Omega} \leq c\|f\|_{1, p(\cdot), \Omega}, \quad \frac{1}{q(x)}=\frac{1}{p(x)}-\frac{\lambda(x)}{n},
$$

that theorem being applicable since

$$
\sup _{x \in \Omega} \lambda(x) p(x)<\sup _{x \in \Omega}[1-\alpha(x)] p(x)<n
$$

according to (19) and (16).

Thus the boundedness of $\mathcal{D}^{\alpha(\cdot)}$ from $W^{1, p(\cdot)}(\Omega)$ into $L^{q(\cdot)}(\Omega)$ has been proved for $q(x)$ of the form (18). The equivalence of (18) to (17) may be directly verified.

For constant exponents the following statement holds.

Corollary 5.2. Let $\alpha$ and $\Omega$ be as in Theorem 5.1 and suppose that $1<p<$ $\frac{n}{1-\alpha}$. Then there exists $c>0$ such that

$$
\left\|\mathcal{D}^{\alpha} f\right\|_{q, \Omega} \leq c\|f\|_{1, p, \Omega}, \quad f \in W^{1, p}(\Omega),
$$

for any exponent $q$ fulfilling

$$
p \leq q<\frac{n p}{n-(1-\alpha) p} .
$$

Theorem 4.4 allow us to conclude about the pointwise convergence of the hypersingular integral. More precisely, the following statement may be derived.

Proposition 5.3. Let $\Omega$ be a bounded open set with Lipschitz boundary. Under the assumption (2) on $p(\cdot)$, the hypersingular integral $\mathcal{D}^{\alpha(\cdot)}$, with $0<\alpha_{0} \leq$ $\alpha(x)<1, x \in \Omega$, of functions in $W^{1, p(\cdot)}(\Omega)$ converges at all those points $x \in \bar{\Omega}$ for which $p(x)(1-\alpha(x))>n$.

Proof. The pointwise convergence of the hypersingular integral is an immediate consequence of (9). We only observe that the assumption $p(x)(1-\alpha(x))>n$ implies $\inf _{x \in \Omega} p(x)>n$. 
Acknowledgement. The first author has been partially supported by Unidade de Investigação "Matemática e Aplicações" of Universidade de Aveiro, through Programa Operacional "Ciência, Tecnologia e Inovação" (POCTI) of the Fundação para a Ciência e a Tecnologia (FCT), co-financed by the European Community fund FEDER.

\section{References}

[1] Adams, D. and Hedberg, L. I., Function Spaces and Potential Theory. Berlin: Springer 1996.

[2] Almeida, A., Inversion of the Riesz potential operator on Lebesgue spaces with variable exponent. Fract. Calc. Appl. Anal. 6 (2003)(3), $311-327$.

[3] Almeida, A. and Samko, S., Characterization of Riesz and Bessel potentials on variable Lebesgue spaces. J. Funct. Spaces Appl. 4 (2006)(2), 113 - 144.

[4] Bojarski, B. and Hajłasz, P., Pointwise inequalities for Sobolev functions and some applications. Studia Math. 106 (1993)(1), 77 - 92.

[5] Capone, C., Cruz-Uribe, D. and Fiorenza, A., The fractional maximal operator on variable $L^{p}$ spaces. Rev. Mat. Iberoamericana (to appear).

[6] Cruz-Uribe, D., Fiorenza, A., Martell, J. M. and Pérez, C., The boundedness of classic operators on variable $L^{p}$ spaces. Ann. Acad. Sci. Fenn. Math. 31 (2006), $239-264$.

[7] Cruz-Uribe, D., Fiorenza, A. and Neugebauer, C. J., The maximal function on variable $L^{p}$ spaces. Ann. Acad. Sci. Fenn. Math. 28 (2003), $223-238$.

[8] Diening, L., Maximal function on generalized Lebesgue spaces $L^{p(\cdot)}$. Math. Inequal. Appl. 7 (2004)(2), $245-253$.

[9] Diening, L., Maximal function on Musiełak-Orlicz spaces and generalized Lebesgue spaces. Bull. Sci. Mat. 129 (2005), $657-700$.

[10] Diening, L., Riesz potential and Sobolev embeddings on generalized Lebesgue and Sobolev spaces $L^{p(\cdot)}$ and $W^{k, p(\cdot)}$. Math. Nachr. 268 (2004), $31-43$.

[11] Diening, L. and Růžička, M., Calderón-Zygmund operators on generalized Lebesgue spaces $L^{p(\cdot)}$ and problems related to fluid dynamics. J. Reine Angew. Math. 563 (2003), 197 - 220.

[12] Edmunds, D. E. and Rákosník, J., Density of smooth functions in $W^{k, p(x)}(\Omega)$. Proc. Roy. Soc. London Ser. A 437 (1992), 229 - 236.

[13] Edmunds, D. E. and Rákosník, J., Sobolev embeddings with variable exponent. Stud. Math. 143 (2000)(3), 267 - 293.

[14] Edmunds, D. E. and Rákosník, J., Sobolev embeddings with variable exponent II. Math. Nachr. 246/247 (2002), 53 - 67.

[15] Fan, X., Shen, J. and Zhao, D., Sobolev embedding theorems for spaces $W^{k, p(x)}(\Omega)$. J. Math. Anal. Appl. 262 (2001)(2), $749-760$. 
[16] Fan, X. and Zhao, D., On the spaces $L^{p(x)}(\Omega)$ and $W^{m, p(x)}(\Omega)$. J. Math. Anal. Appl. 263 (2001)(2), $424-446$.

[17] Fiorenza, A., A mean continuity type result for certain Sobolev spaces with variable exponent. Commun. Contemp. Math. 4 (2002)(3), 587 - 605.

[18] Gilbarg, D. and Trudinger, N. S., Elliptic Partial Differential Equations of Second Order. Berlin: Springer 2001.

[19] Hajłasz, P., Boundary behaviour of Sobolev mappings. Proc. Amer. Math. Soc. 123 (1995)(4), 1145 - 1148.

[20] Hajłasz, P. and Martio, O., Traces of Sobolev functions on fractal type sets and characterization of extension domains. J. Funct. Anal. 143 (1997), $221-246$.

[21] Harjulehto, P. and Hästö, P., A capacity approach to the Poincaré inequality and Sobolev imbeddings in variable exponent Sobolev spaces. Rev. Mat. Complut. 17 (2004)(1), 129 - 146.

[22] Karapetyants, N. K. and Ginzburg, A. I., Fractional integrodifferentiation in Hölder classes of arbitrary order. Georgian Math. J. 2 (1995)(2), 141 - 150.

[23] Kinnunen, J. and Martio, O., Hardy's inequalities for Sobolev functions. Math. Res. Lett. 4 (1997)(4), $489-500$.

[24] Kokilashvili, V. and Samko, S., On Sobolev theorem for Riesz-type potentials in Lebesgue spaces with variable exponent. Z. Anal. Anwendungen 22 (2003)(4), $899-910$.

[25] Kokilashvili, V. and Samko, S., Singular integrals in weighted Lebesgue spaces with variable exponent. Georgian Math. J. 10 (2003)(1), 145 - 156.

[26] Kokilashvili, V. and Samko, S., Maximal and fractional operators in weighted $L^{p(x)}$ spaces. Rev. Mat. Iberoamericana 20 (2004), 493 - 515.

[27] Kovácik, O. and Rákosník, J., On spaces $L^{p(x)}$ and $W^{k, p(x)}$. Czech. Math. J. 41(416) (1991)(4), $592-618$.

[28] Levine, S., Stanich, J. and Chen, Y., Image restoration via nonstandard diffusion. Technical Report 04-01 (2004), Dept. Math. Computer Science, Duquesne University.

[29] Nekvinda, A., Hardy-Littlewood maximal operator on $L^{p(x)}\left(\mathbb{R}^{n}\right)$. Math. Inequal. Appl. 7 (2004)(2), 255 - 265.

[30] Rákosník, J., Sobolev inequality with variable exponent. In: Function Spaces, Differential Operators and Nonlinear Analysis (eds.: V. Mustonen et al.). Prague: Math. Institute, Acad. Sci. Czech Republic 2000, pp. 220 - 228.

[31] Ross, B. and Samko, S., Fractional integration operator of variable order in the Hölder spaces $H^{\lambda(x)}$. Internat. J. Math. Sci. 18 (1995)(4), $777-788$.

[32] Růžička, M., Electrorheological Fluids: Modeling and Mathematical Theory. Lect. Notes Math. 1748. Berlin: Springer 2000. 
[33] Samko, S., Denseness of $C_{0}^{\infty}\left(\mathbb{R}^{N}\right)$ in the generalized Sobolev spaces $W^{M, P(X)}\left(\mathbb{R}^{N}\right)$. In: Direct and Inverse Problems of Mathematical Physics (ISAAC'97; ed.: R. P. Gilbert). Dordrecht: Kluwer 2000, Int. Soc. Anal. Appl. Comput. 5, pp. $333-342$.

[34] Samko, S. G., Convolution and potential type operators in $L^{p(x)}\left(\mathbb{R}^{n}\right)$. Integral Transforms Spec. Funct. 7 (1998)(3-4), $261-284$.

[35] Samko, S. G., Convolution type operators in $L^{p(x)}$. Integral Transforms Spec. Funct. 7 (1998)(1-2), 123 - 144.

[36] Samko, S. G., Hypersingular Integrals and their Applications. London: Taylor \& Francis 2002.

[37] Samko, S. G., On a progress in the theory of Lebesgue spaces with variable exponent: maximal and singular operators. Integral Transforms Spec. Funct. 16 (2005)(5-6), $461-482$.

[38] Sharapudinov, I. I., Topology of the space $\mathcal{L}^{p(t)}([0,1])$. Math. Notes 26 (1979), $796-806$.

[39] Stein, E. M., Singular Integrals and Differentiability Properties of Functions. Princeton: University Press 1970.

Received May 19, 2005; revised December 5, 2005 